\title{
ASYMPTOTIC AXISYMMETRY OF THE SUBSONIC TRAVELING WAVES TO THE GROSS-PITAEVSKII EQUATION
}

\author{
JUNCHENG WEI AND WEI YAO
}

\begin{abstract}
In this note we show the asymptotic axisymmetry of subsonic traveling waves of finite energy to the Gross-Pitaevskii equation. In particular, using a new Hamiltonian identity, we prove that the momentum in the orthogonal directions must vanish.
\end{abstract}

\section{INTRODUCTION}

This note is concerned with the traveling wave solutions to the Gross-Pitaevskii equation

$$
i \partial_{t} \Psi=\Delta \Psi+\Psi\left(1-|\Psi|^{2}\right) \quad \text { for }(t, x) \in \mathbb{R} \times \mathbb{R}^{N},
$$

where $\Psi$ is a complex-valued function. GP equation appears in many physical problems, such as: Bose-Einstein condensation, superconductivity, nonlinear optics, etc. We refer the reader to [4 and the references therein for more details about this subject.

On a formal level, the Gross-Pitaevskii equation is Hamiltonian. The two important conserved Hamiltonians are: the so-called Ginzburg-Landau energy

$$
E(\Psi)=\frac{1}{2} \int_{\mathbb{R}^{N}}|\nabla \Psi|^{2}+\frac{1}{4} \int_{\mathbb{R}^{N}}\left(1-|\Psi|^{2}\right)^{2}
$$

and the momentum (up to a multiplicative constant of modulus one)

$$
\vec{P}(\Psi)=\frac{1}{2} \int_{\mathbb{R}^{N}}(i \nabla \Psi) \cdot(\Psi-1),
$$

which play an important role in the asymptotic description of traveling waves of finite energy.

If $\Psi$ does not vanish, by using the Madelung transform, one may write

$$
\Psi=\sqrt{\rho} \exp (i \varphi), \quad v=-2 \nabla \varphi .
$$

This leads to the hydrodynamic form of the equation

$$
\left\{\begin{array}{l}
\partial_{t} \rho+\operatorname{div}(\rho v)=0, \\
\rho\left(\partial_{t} v+v \cdot \nabla v\right)+\nabla \rho^{2}=\rho \nabla\left(\frac{\Delta \rho}{\rho}-\frac{|\nabla \rho|^{2}}{2 \rho^{2}}\right) .
\end{array}\right.
$$

If one neglects the right-hand side of the second equation, which is often referred to as the quantum pressure, system (3) is similar to the Euler equation for a compressible fluid, with pressure law $p(\rho)=\rho^{2}$. In particular, the speed of sound waves near the constant solution $\Psi=1$ is given by

$$
c_{s}=\sqrt{2} .
$$


A traveling wave $v$ for the Gross-Pitaevskii equation is a particular solution of equation $\mathrm{GP}$ of the form:

$$
\Psi(t, x)=v\left(x_{1}-c t, x^{\prime}\right),
$$

where $x^{\prime}=\left(x_{2}, \ldots, x_{N}\right)$ denotes the vector of the last $N-1$ variables of $x$ in $\mathbb{R}^{N}$. The parameter $c \in \mathbb{R}$ is the speed of the traveling wave, which moves in direction $x_{1}$ (we may restrict to the case $c \geq 0$ using complex conjugation). The equation for the profile $v$, which we will consider in the following, is given by

$$
i c \partial_{x_{1}} v+\Delta v+v\left(1-|v|^{2}\right)=0, x \in \mathbb{R}^{N} .
$$

We note that, traveling waves conserve the Ginzburg-Landau energy and the momentum, namely: for each fixed $t \in \mathbb{R}$,

$$
\begin{aligned}
E(\Psi(t, \cdot)) & :=\frac{1}{2} \int_{\mathbb{R}^{N}}|\nabla \Psi|^{2}+\frac{1}{4} \int_{\mathbb{R}^{N}}\left(1-|\Psi|^{2}\right)^{2} \\
& =\frac{1}{2} \int_{\mathbb{R}^{N}}|\nabla v|^{2}+\frac{1}{4} \int_{\mathbb{R}^{N}}\left(1-|v|^{2}\right)^{2}=: E(v)
\end{aligned}
$$

and

$$
\begin{aligned}
\vec{P}(\Psi(t, \cdot)) & :=\frac{1}{2} \int_{\mathbb{R}^{N}}(i \nabla \Psi) \cdot(\Psi-1) \\
& =\frac{1}{2} \int_{\mathbb{R}^{N}}(i \nabla v) \cdot(v-1)=: \vec{P}(v) .
\end{aligned}
$$

We will denote the scalar momentum in direction $x_{1}$ (up to a multiplicative constant of modulus one) by

$$
p(v)=\frac{1}{2} \int_{\mathbb{R}^{N}}(i \nabla v) \cdot(v-1) .
$$

In this note we only consider the traveling waves of finite energy, i.e.,

$$
E(v)=\frac{1}{2} \int_{\mathbb{R}^{N}}|\nabla v|^{2}+\frac{1}{4} \int_{\mathbb{R}^{N}}\left(1-|v|^{2}\right)^{2}<+\infty .
$$

The traveling waves of finite energy play an important role in the long time dynamics of the Gross-Pitaevskii equation. Whereas the one-dimensional case can be integrated explicitly, solutions are not explicitly known in higher dimensions. The first attempts to prove existence of traveling waves and to study their properties were based on formal expansions and numerics. Among them, Jones, Putterman and Roberts [14, 15] developed a thorough analysis in dimensions two and three: they found a branch of traveling waves with speeds $c$ in the full subsonic range $(0, \sqrt{2})$, and they conjectured that there exist non-constant traveling waves of finite energy if and only if their speed $c$ is in the subsonic range.

More recently, the program of Jones, Putterman and Roberts [14, 15] has been provided with rigorous mathematical proofs (see the article [4] to review some of these progresses). For example, the non-existence of non-constant supersonic traveling waves (i.e., $c>\sqrt{2}$ ) of finite energy in dimension $N \geq 2$ in [9], the non-existence of non-constant sonic traveling waves (i.e., $c=\sqrt{2}$ ) of finite energy in dimension two in [11] and the existence of non-constant axisymmetric traveling waves in [2, 1, 3, 5, 17, 16. However, to our knowledge, their conjecture remains open. A number of open problems is also stressed in [4, such as existence of traveling waves of finite energy to (TWc for all values of speeds $0<c<\sqrt{2}$ in dimension two, and non-existence of sonic traveling waves of finite energy in 
dimension three. To solve these problems the study of asymptotic behaviours of traveling waves plays an important role. As showed in [10] and [11] the asymptotic behaviour of sonic traveling waves is much involved than in the subsonic case. In the following we only consider subsonic traveling waves of finite energy.

For subsonic traveling waves, i.e., $0<c<\sqrt{2}$, Jones, Putterman and Roberts [14, 15] did some formal computations and described the asymptotic behaviour of traveling waves of finite energy which are axisymmetric around axis $x_{1}$. They computed their first order asymptotics (up to a multiplicative constant of modulus one) in dimension two:

$$
v(x)-1 \sim \frac{i \alpha x_{1}}{x_{1}^{2}+\left(1-\frac{c^{2}}{2}\right) x_{2}^{2}}, \quad \text { as }|x| \rightarrow+\infty,
$$

and in dimension three:

$$
v(x)-1 \sim \frac{i \alpha x_{1}}{\left(x_{1}^{2}+\left(1-\frac{c^{2}}{2}\right)\left(x_{2}^{2}+x_{3}^{2}\right)\right)^{3 / 2}}, \quad \text { as }|x| \rightarrow+\infty .
$$

Here, the real constant $\alpha$ is the stretched dipole coefficient linked to the GinzburgLandau energy $E(v)$ and to the scalar momentum $p(v)$, by the formulae

$$
2 \pi \alpha \sqrt{1-\frac{c^{2}}{2}}=c E(v)+2\left(1-\frac{c^{2}}{4}\right) p(v)
$$

in dimension two, and

$$
4 \pi \alpha=\frac{c}{2} E(v)+2 p(v)
$$

in dimension three.

Recently Gravejat [12] proved rigorously formulae (7), (8), (9) and $(10)$ in the axisymmetric case or in dimension two. Later on he [13, gave their first order asymptotics at infinity in the general case, which is the start point of our result.

Theorem A ([13]). Let $N \geq 2$ and $0<c<\sqrt{2}$. Consider a travelling wave $v$ of finite energy and of speed $c$ for equation (TWc). There exists a complex number $\lambda_{\infty}$ of modulus one and a smooth function $v_{\infty}$ defined from the sphere $\mathbb{S}^{N-1}$ to $\mathbb{R}$ such that

$$
|x|^{N-1}\left(v(x)-\lambda_{\infty}\right)-i \lambda_{\infty} v_{\infty}\left(\frac{x}{|x|}\right) \rightarrow 0 \quad \text { uniformly as }|x| \rightarrow+\infty .
$$

Moreover, the function $v_{\infty}$ is given by

$$
v_{\infty}(\sigma)=\alpha \frac{\sigma_{1}}{\left(1-\frac{c^{2}}{2}+\frac{c^{2} \sigma_{1}^{2}}{2}\right)^{\frac{N}{2}}}+\sum_{j=2}^{N} \beta_{j} \frac{\sigma_{j}}{\left(1-\frac{c^{2}}{2}+\frac{c^{2} \sigma_{1}^{2}}{2}\right)^{\frac{N}{2}}}, \quad \forall \sigma \in \mathbb{S}^{N-1},
$$

where the constants $\alpha$ and $\beta_{j}$ are equal to

$$
\begin{aligned}
\alpha & =\frac{\Gamma\left(\frac{N}{2}\right)}{2 \pi^{\frac{N}{2}}}\left(1-\frac{c^{2}}{2}\right)^{\frac{N-3}{2}}\left(\frac{4-N}{2} c E(v)+\left(2+\frac{N-3}{2} c^{2}\right) p(v)\right), \\
\beta_{j} & =\frac{\Gamma\left(\frac{N}{2}\right)}{\pi^{\frac{N}{2}}}\left(1-\frac{c^{2}}{2}\right)^{\frac{N-1}{2}} P_{j}(v), \quad \text { for } 2 \leq j \leq N .
\end{aligned}
$$

Remark 1. In a previous paper 12 Gravejat has conjectured that function $v_{\infty}$ will have the form (12) (see Conjecture 1 of [12]) and proved it in dimension two and in the axisymmetric case. 
Remark 2. The study of the asymptotic behaviour of sonic traveling waves is much more involved than in the subsonic case. To our knowledge, the known result on the asymptotic behaviour of sonic traveling waves is their convergence at infinity towards a constant of modulus one in [11].

Remark 3. If a traveling wave $v$ is axisymmetric around axis $x_{1}$, then it is an even function of each variable $x_{j}$ for $2 \leq j \leq N$. Therefore, the functions $v$ and $\partial_{x_{j}} v$ are respectively even and odd functions of each variable $x_{j}$. By definition (5), the scalar momentum $P_{j}(v)$, and consequently the constant $\beta_{j}$, vanish for every $2 \leq j \leq N$.

Remark 4. By the proof of Corollary 1 in [12, the stretched dipole coefficient $\alpha$ given in $(13)$ is non-negative.

Remark 5. There is an interesting question raised in the Remark 1 of 12 or Remark 3 of [13]: Is it possible to construct travelling waves $v$ whose asymptotic behaviour correspond to any possible one given by Theorem $A$, or are there other restrictions for the possible asymptotic behaviours? Indeed, the existence of presumably axisymmetric traveling waves, for which the constants $\beta_{j}=0$ for all $2 \leq j \leq N$, has been proved in Béthuel and Saut [2, Béthuel, Gravejat and Saut [3], Béthuel, Orlandi and Smets [1, Chiron [5] and Mariş [17] by using variational method, and in Lin and Wei [16] by using a perturbative approach.

In this note we give an answer to the above question by proving that the constants $\beta_{j}=0,2 \leq j \leq N$, for all subsonic traveling waves of finite energy. This fact also implies that any subsonic traveling wave of finite energy is asymptotic axisymmetric. More precisely, we have the following result:

Theorem 1.1. Let $N \geq 2$ and $0<c<\sqrt{2}$. Then for any traveling wave $v$ of finite energy and of speed $c$ for equation (TWc),

$$
P_{j}(v)=0, \quad 2 \leq j \leq N .
$$

As a consequence, $v$ is asymptotically axisymmetric around axis $x_{1}$. Namely, there exists a complex number $\lambda_{\infty}$ of modulus one and a real number $\alpha$ such that

$$
|x|^{N-1}\left[v(x)-\lambda_{\infty}-\lambda_{\infty} \frac{i \alpha x_{1}}{\left(x_{1}^{2}+\left(1-\frac{c^{2}}{2}\right)\left|x^{\prime}\right|^{2}\right)^{N / 2}}\right] \rightarrow 0
$$

uniformly as $|x| \rightarrow+\infty$, where $x^{\prime}=\left(x_{2}, \ldots, x_{N}\right)$ denotes the vector of the last $N-1$ variables of $x$ in $\mathbb{R}^{N}$. Moreover, the constant $\alpha$ is non-negative and equals to

$$
\alpha=\frac{\Gamma\left(\frac{N}{2}\right)}{2 \pi^{\frac{N}{2}}}\left(1-\frac{c^{2}}{2}\right)^{\frac{N-3}{2}}\left(\frac{4-N}{2} c E(v)+\left(2+\frac{N-3}{2} c^{2}\right) p(v)\right) .
$$

Remark 6. Although the asymptotic axisymmetry of the traveling waves is proved in the above theorem, we do not know whether any traveling wave is axisymmetric. This raises an interesting question: does there exist non-axisymmetric traveling wave to the Gross-Pitaevskii equation?

Remark 7. In the following, we will always assume that

$$
\lambda_{\infty}=1 \text {. }
$$

Indeed, if this is not the case, we can study the function $\lambda_{\infty}^{-1} v$ instead of $v$ because it is also a traveling wave of finite energy and of speed $c$ satisfying equation (TWc). 
As mentioned in [12, one of the main interests of Theorem $\mathrm{A}$ and 1.1 is the sharpness of the decay exponent $N-1$ at infinity they give. Actually, one can show that any subsonic traveling wave $v$ such that the function

$$
x \mapsto|x|^{\sigma}(v(x)-1)
$$

is bounded on $\mathbb{R}^{N}$ for some $\sigma>N-1$, must be a constant traveling wave by applying the following corollary of Theorem 1.1 .

Corollary 1.2. Let $N \geq 2$ and $0<c<\sqrt{2}$. Consider a traveling wave $v$ for the Gross-Pitaevskii equation of finite energy and of speed $c$. Then, the constant $\alpha$ in formula (17) is equal to 0 if and only if $v$ is a constant traveling wave.

It is easily seen that the following result is another application of Theorem 1.1 .

Corollary 1.3. Assume $N=2$ and $0<c<\sqrt{2}$. Consider a non-constant traveling wave $v$ of finite energy and of speed $c$ for the Gross-Pitaevskii equation. Then, the function $v-1$ does not belong to $L^{2}\left(\mathbb{R}^{2}\right)$. In particular, it does not belong to $H^{1}\left(\mathbb{R}^{2}\right)$.

This last observation plays an important role in the variational argument of [3] for the construction of finite energy solutions to (TWc). It is proved in Corollary 2 of 4 in the axisymmetric case.

The proof of Corollary 1.2 is similar to the arguments in the proof of Corollary 1 in [12]. The only difference is that in [12] the axisymmetric condition on $v$ is assumed, but here we can remove this condition by Theorem 1.1. So we omit its proof here.

This note will be organized around the proof of Theorem 1.1. In Section 2, we introduce the Sobolev regularity and algebraic decay of subsonic traveling waves. In Section 3, we calculate a new Hamiltonian integral quantity for subsonic traveling waves to prove the scalar momentum $P_{j}(v)=0$ for all $2 \leq j \leq N$ and complete the proof of Theorem 1.1 .

\section{Preliminaries}

In this section we recall some regularity and decay estimates of subsonic traveling waves of finite energy to equation (TWc), which will be used in the calculation of a new Hamiltonian integral quantity in the next section.

The first result is the local smoothness and the Sobolev regularity of subsonic traveling waves given in [10], see also the articles of F. Béthuel and J.C. Saut [2], of Béthuel, Gravejat and Saut [3] and of A. Farina [6].

Proposition $2.1(10)$. If $v$ is a subsonic traveling wave of finite energy of equation (TWc in $L_{l o c}^{1}\left(\mathbb{R}^{N}\right)$, then, $v$ is $C^{\infty}$, bounded, and the functions $\eta:=1-|v|^{2}$ and $\nabla v$ belong to all the spaces $W^{k, p}\left(\mathbb{R}^{N}\right)$ for $k \in \mathbb{N}$ and $1<p \leq \infty$.

It follows that the modulus $\rho$ of $v$ converges to 1 at infinity. In particular, there is some $R_{0}>0$ such that

$$
\rho \geq \frac{1}{2} \quad \text { on }|x| \geq R_{0}
$$

Since the Ginzburg-Landau energy $E(v)$ is finite, there is a lifting $\theta$ of $v$ on $B\left(0, R_{0}\right)^{c}$ such that

$$
v=\rho e^{i \theta} .
$$


As in [12] we introduce a cut-off function $\psi \in C^{\infty}\left(\mathbb{R}^{N},[0,1]\right)$ such that

$$
\begin{cases}\psi=0 & \text { on } B\left(0,2 R_{0}\right) \\ \psi=1 & \text { on } B\left(0,3 R_{0}\right)^{c} .\end{cases}
$$

Then all the asymptotic estimates obtained in [10] are independent of the choice of $R_{0}$ and $\psi$. In particular, we have the following algebraic decay for the functions $\eta$ and $\nabla(\psi \theta)$ given in [10, 12.

Proposition $2.2([10,12])$. The functions $\eta, \nabla \eta$ and $\nabla(\psi \theta)$ belong to respectively $M_{N}^{\infty}\left(\mathbb{R}^{N}\right), M_{N+1}^{\infty}\left(\mathbb{R}^{N}\right)$ and $M_{N}^{\infty}\left(\mathbb{R}^{N}\right)$. Here the space $M_{\delta}^{\infty}\left(\mathbb{R}^{N}\right)$ is defined by

$$
M_{\delta}^{\infty}\left(\mathbb{R}^{N}\right)=\left\{u: \mathbb{R}^{N} \rightarrow \mathbb{C} \mid\|u\|_{M_{\delta}^{\infty}\left(\mathbb{R}^{N}\right)}=\sup \left\{|x|^{\delta}|u(x)|, x \in \mathbb{R}^{N}\right\}<+\infty\right\} .
$$

The proofs of Theorem A Proposition 2.1 and Proposition 2.2 rely on a new formulation of equation (TWC and the use of convolution equations, in particular on a careful analysis of the kernels they involve. For the details, we refer the reader to [10, 12].

\section{Proof of Theorem 1.1}

Proof of Theorem 1.1. It is sufficient to prove that $P_{j}(v)=0$ for all $2 \leq j \leq N$. First of all, by Proposition 2.2 the momentum $\vec{P}(v)$ in (5) is well-defined. Secondly for $2 \leq j \leq N$ we define a new Hamiltonian integral quantity:

$$
E_{j}\left(v, x_{1}\right):=\int_{\mathbb{R}^{N-1}} x_{j}\left[\frac{1}{2}\left(\left|\nabla_{x^{\prime}} v\right|^{2}-\left|\partial_{x_{1}} v\right|^{2}\right)+\frac{1}{4}\left(1-|v|^{2}\right)^{2}\right] d x^{\prime}
$$

which was also used in 8 to show the axial symmetry of steady state solutions of nonlinear Schrödinger equations. (This type of Hamiltonian identities was due to Gui [7.) By Proposition 2.2 it is well-defined for any $x_{1} \in \mathbb{R}$.

By Proposition 2.1. using integration by parts and equation (TWc), we can get

$$
\begin{aligned}
\frac{d E_{j}}{d x_{1}} & =\int_{\mathbb{R}^{N-1}} x_{j}\left[\nabla_{x^{\prime}} v \cdot \nabla_{x^{\prime}} \partial_{x_{1}} v-\partial_{x_{1}} v \cdot \partial_{x_{1}}^{2} v-v\left(1-|v|^{2}\right) \cdot \partial_{x_{1}} v\right] d x^{\prime} \\
& =\int_{\mathbb{R}^{N-1}} x_{j}\left[\nabla_{x^{\prime}} v \cdot \nabla_{x^{\prime}} \partial_{x_{1}} v+\partial_{x_{1}} v \cdot \Delta_{x^{\prime}} v\right] d x^{\prime} \\
& =-\int_{\mathbb{R}^{N-1}} \partial_{x_{j}} v \cdot \partial_{x_{1}} v d x^{\prime}=:-H_{j}\left(v, x_{1}\right) .
\end{aligned}
$$

Here, for $j=2, \ldots, N$, we define

$$
H_{j}\left(v, x_{1}\right):=\int_{\mathbb{R}^{N-1}} \partial_{x_{j}} v \cdot \partial_{x_{1}} v d x^{\prime}
$$


Applying Proposition 2.2 again, $H_{j}\left(v, x_{1}\right)$ is well-defined for all $x_{1} \in \mathbb{R}$. By Proposition 2.1. using integration by parts and equation (TWC, one can get

$$
\begin{aligned}
\frac{d H_{j}}{d x_{1}}= & \int_{\mathbb{R}^{N-1}}\left[\partial_{x_{1}} \partial_{x_{j}} v \cdot \partial_{x_{1}} v+\partial_{x_{j}} v \cdot \partial_{x_{1}}^{2} v\right] d x^{\prime} \\
= & \int_{\mathbb{R}^{N-1}} \partial_{x_{j}} \partial_{x_{1}} v \cdot \partial_{x_{1}} v d x^{\prime}-\sum_{k=2}^{N} \int_{\mathbb{R}^{N-1}} \partial_{x_{j}} v \cdot \partial_{x_{k}}^{2} v d x^{\prime} \\
& -c \int_{\mathbb{R}^{N-1}} \partial_{x_{j}} v \cdot\left(i \partial_{x_{1}} v\right) d x^{\prime}-\int_{\mathbb{R}^{N-1}} \partial_{x_{j}} v \cdot v\left(1-|v|^{2}\right) d x^{\prime} \\
= & \int_{\mathbb{R}^{N-1}} \partial_{x_{j}} \partial_{x_{1}} v \cdot \partial_{x_{1}} v d x^{\prime}+\sum_{k=2}^{N} \int_{\mathbb{R}^{N-1}} \partial_{x_{j}} \partial_{x_{k}} v \cdot \partial_{x_{k}} v d x^{\prime} \\
& -c \int_{\mathbb{R}^{N-1}} \partial_{x_{j}} v \cdot\left(i \partial_{x_{1}} v\right) d x^{\prime}-\int_{\mathbb{R}^{N-1}} \partial_{x_{j}} v \cdot v\left(1-|v|^{2}\right) d x^{\prime} \\
= & \frac{1}{2} \sum_{k=1}^{N} \int_{\mathbb{R}^{N-1}} \partial_{x_{j}}\left|\partial_{x_{k}} v\right|^{2} d x^{\prime}-c \int_{\mathbb{R}^{N-1}} \partial_{x_{j}} v \cdot\left(i \partial_{x_{1}} v\right) d x^{\prime} \\
& +\frac{1}{4} \int_{\mathbb{R}^{N-1}} \partial_{x_{j}}\left(1-|v|^{2}\right)^{2} d x^{\prime} \\
= & -c \int_{\mathbb{R}^{N-1}} \partial_{x_{j}} v \cdot\left(i \partial_{x_{1}} v\right) d x^{\prime} .
\end{aligned}
$$

Note that

$$
2 \partial_{x_{j}} v \cdot\left(i \partial_{x_{1}} v\right)=\partial_{x_{j}}\left((v-1) \cdot i \partial_{x_{1}} v\right)+\partial_{x_{1}}\left(\partial_{x_{j}} v \cdot i(v-1)\right) .
$$

Hence

$$
\begin{aligned}
\frac{d H_{j}}{d x_{1}} & =-\frac{c}{2} \int_{\mathbb{R}^{N-1}}\left[\partial_{x_{j}}\left((v-1) \cdot i \partial_{x_{1}} v\right)+\partial_{x_{1}}\left(\partial_{x_{j}} v \cdot i(v-1)\right)\right] d x^{\prime} \\
& =-\frac{c}{2} \int_{\mathbb{R}^{N-1}} \partial_{x_{1}}\left(\partial_{x_{j}} v \cdot i(v-1)\right) d x^{\prime} \\
& =-\frac{c}{2} \frac{d}{d x_{1}} \int_{\mathbb{R}^{N-1}} \partial_{x_{j}} v \cdot i(v-1) d x^{\prime}
\end{aligned}
$$

By the decay estimates in Proposition 2.2 we get

$$
H_{j}\left(v, x_{1}\right)=-\frac{c}{2} \int_{\mathbb{R}^{N-1}} \partial_{x_{j}} v \cdot i(v-1) d x^{\prime}=\frac{c}{2} \int_{\mathbb{R}^{N-1}} i \partial_{x_{j}} v \cdot(v-1) d x^{\prime} .
$$

Then, by (19) and 24) we have

$$
\frac{d E_{j}}{d x_{1}}=-\frac{c}{2} \int_{\mathbb{R}^{N-1}} i \partial_{x_{j}} v \cdot(v-1) d x^{\prime}
$$

Integrating the above equality on $\mathbb{R}$ and applying Proposition 2.2 , we get

$$
c P_{j}=\frac{c}{2} \int_{\mathbb{R}^{N}} i \partial_{x_{j}} v \cdot(v-1) d x=-\int_{-\infty}^{+\infty} \frac{d E_{j}}{d x_{1}} d x_{1}=0 .
$$

Therefore, we get $\beta_{j}=0$ for all $2 \leq j \leq N$ by formula (14) and complete the proof. 
Acknowledgments: The research of the first author is partially supported by an Earmarked Grant from RGC of Hong Kong. The authors would like to thank the referee for several helpful comments concerning the exposition of the paper, especially for drawing the authors' attention to [17].

\section{REFERENCES}

[1] F. Béthuel, G. Orlandi, and D. Smets, Vortex rings for the Gross-Pitaevskii equation, J. Eur. Math. Soc., 6(1), 17-94, 2004.

[2] F. Béthuel and J.-C. Saut, Travelling waves for the Gross-Pitaevskii equation. I, Ann. Inst. Henri Poincaré, Physique Théorique, 70(2), 147-238, 1999.

[3] F. Béthuel, P. Gravejat and J.-C. Saut, Travelling waves for the Gross-Pitaevskii equation. II, Comm. Math. Phys., 285(2), 567-651, 2009.

[4] F. Béthuel, P. Gravejat, and J.-C. Saut, Existence and properties of travelling waves for the Gross-Pitaevskii equation, in "Stationary and Time Dependent Gross-Pitaevskii Equations", A. Farina and J.-C. Saut editors, Contemporary Mathematics, 473, 55-104, American Mathematical Society, Providence, R.I., 2008.

[5] D. Chiron, Travelling waves for the Gross-Pitaevskii equation in dimension larger than two, Nonlinear Anal., 58(1-2), 175-204, 2004.

[6] A. Farina, From Ginzburg-Landau to Gross-Pitaevskii, Monatsh. Math., 139, 265-269, 2003.

[7] C. Gui, Halmitonian identities for elliptic partial differential equations, J. Funct. Anal., 254(4), 904-933, 2008.

[8] C. Gui, A. Malchiodi, and H. Xu, Axial of symmetry of stationary solutions to super critical nonlinear Schrödinger equation, Proc. Amer. Math. Soc., 139(3), 1023-1032, 2011.

[9] P. Gravejat, A non-existence result for supersonic travelling waves in the Gross-Pitaevskii equation, Commun, Math, Phys., 243(1), 93-103, 2003.

[10] P. Gravejat, Decay for travelling waves in the Gross-Pitaevskii equation, Ann. Inst. Henri Poincaré, Analyse Non Linéaire, 21(5), 591-637, 2004.

[11] P. Gravejat, Limit at infinity and nonexistence results for sonic travelling waves in the Gross-Pitaevskii equation, Differential Integral Equations, 17(11-12), 1213-1232, 2004.

[12] P. Gravejat, Asymptotics for the travelling waves in the Gross-Pitaevskii equation, Asymptot. Anal., 45(3-4), 227-299, 2005.

[13] P. Gravejat, First order asymptotics for the travelling waves in the Gross-Pitaevskii equation, Adv. Differential Equations, 11(3), 259-280, 2006.

[14] C.A. Jones, S.J. Putterman, and P.H. Roberts, Motions in a Bose condensate V. Stability of solitary wave solutions of nonlinear Schrödinger equations in two and three dimensions, J. Phys. A, Math. Gen., 19, 2991-3001, 1986.

[15] C.A. Jones, P.H. Roberts, Motions in a Bose condensate IV. Axisymmetric solitary waves, J. Phys. A, Math. Gen., 15, 2599-2619, 1982.

[16] F.-H. Lin, J.-C. Wei, Traveling wave solutions of the Schrödinger map equation, Comm. Pure Appl. Math., 63(12), 1585-1621, 2010.

[17] M. Mariş, Traveling waves for nonlinear Schrödinger equations with nonzero conditions at infinity, Preprint, 2009.

J. Wei - Department of Mathematics, Chinese University of Hong Kong, Shatin, Hong KONG

E-mail address: wei@math.cuhk.edu.hk

W. Yao - Department of Mathematics, Chinese University of Hong Kong, Shatin, HONG KONG

E-mail address: wyao@math.cuhk.edu.hk 